\title{
Outpatient spine surgery: defining the outcomes, value, and barriers to implementation
}

\author{
Arjun Vivek Pendharkar, MD, Maryam Nour Shahin, BS, Allen Lin Ho, MD, \\ Eric Scott Sussman, MD, David Arnold Purger, MD, PhD, Anand Veeravagu, MD, \\ John Kevin Ratliff, MD, and Atman Mukesh Desai, MD \\ Department of Neurosurgery, Stanford University, Stanford, California
}

\begin{abstract}
Spine surgery is a key target for cost reduction within the United States health care system. One possible strategy involves the transition of inpatient surgeries to the ambulatory setting. Lumbar laminectomy with or without discectomy, lumbar fusion, anterior cervical discectomy and fusion, and cervical disc arthroplasty all represent promising candidates for outpatient surgeries in select populations. In this focused review, the authors clarify the different definitions used in studies describing outpatient spine surgery. They also discuss the body of evidence supporting each of these procedures and summarize the proposed cost savings. Finally, they examine several patient- and surgeon-specific considerations to highlight the barriers in translating outpatient spine surgery into actual practice.
\end{abstract}

https://thejns.org/doi/abs/10.3171/2018.2.FOCUS17790

KEYWORDS anterior cervical discectomy and fusion; cervical disc arthroplasty; lumbar laminectomy; transforaminal lumbar interbody fusion; minimally invasive surgery; ambulatory surgery; cost; outcomes; outpatient

$\mathrm{S}$ PINE surgery is one of the most impactful targets for reducing costs within the United States health care system. ${ }^{32}$ An estimated $\$ 90$ billion is spent each year on the diagnosis and management of low-back pain alone..$^{28}$ As summarized by Resnick et al., spinal disorders are an ideal target for cost reduction because of their high prevalence and significant contribution to morbidity- and disability-related costs. ${ }^{36}$ Furthermore, there is significant variability in the treatment paradigms for spinal disorders-representing the entire spectrum of pain medications, acupuncture, massage therapy, steroid injections, surgical decompression and fusion, and beyond. It is the enormous clinical burden of spinal disease paired with treatment heterogeneity that creates an opportunity to empirically define real value and produce savings for the health care system.

One promising but controversial cost reduction strategy involves transitioning surgical procedures to an outpatient setting. More than 54 million outpatient procedures are performed annually in the United States. Among Medicare beneficiaries, rates of outpatient surgery have increased by $40 \%$ in the last 10 years. And the number of ambulatory surgery centers has grown by $60 \%$ within the same time period. ${ }^{21}$ Although eye surgeries, arthroscopic procedures, peripheral nerve cases, and soft tissue cases represent the majority of ambulatory operations, an increasing proportion of spine surgeries has transitioned to the outpatient setting. ${ }^{2,5,7}$ Lumbar laminectomy with or without discectomy, lumbar fusion, anterior cervical discectomy and fusion, and cervical disc arthroplasty all represent promising candidates for outpatient surgeries in select populations.

In this focused review, we clarify the different definitions used in studies describing outpatient spine surgery. We also discuss the body of evidence supporting the transition of each of these procedures to an outpatient setting and summarize the proposed cost savings. Finally, we examine several patient- and surgeon-specific considerations to highlight the barriers in translating outpatient spine surgery into actual practice.

\section{Definitions}

Within the current body of literature describing outpatient spine surgery, there exists a heterogeneous and ill- 
defined set of terms that obscures true understanding of the outcomes and cost savings. Fundamentally, any time a patient is discharged from the hospital and has not been admitted to an inpatient ward, they have undergone an outpatient surgery. However, from a reimbursement perspective, there is a clear delineation between a patient whose recovery is observed within a reasonable amount of time before discharge (for example, 4-6 hours in the Medicare Claims Processing Manual, Chapter 4, Section 290.2.2) and a patient who is observed for an extended period of time $(<24$ hours). Both of these patient encounters, from an outcomes perspective, can be grouped together as an outpatient procedure but carry different hospital utilization costs.

Similarly, utilization differs between an outpatient procedure performed in association with a hospital and one performed at a freestanding ambulatory surgery center. Idowu et al. examined this difference and found that, although there has indeed been a dramatic increase in the number of hospital-associated outpatient spine operations, there has been a significantly less pronounced increase in spine surgery at freestanding ambulatory centers. ${ }^{23} \mathrm{In}$ general, the lack of granularity regarding these definitions represents a significant limitation of the literature describing outpatient outcomes.

\section{Outcomes}

\section{Lumbar Laminectomy and Discectomy}

Lumbar laminectomy with or without discectomy remains the most common spine operation performed in the United States and was one of the earliest procedures to be successfully transitioned to the outpatient setting (Table 1). Several groups have reported clinical series describing favorable outcomes. ${ }^{8,10,22,25,33,47}$ Helseth et al. reported on a series of 1073 consecutive patients undergoing lumbar procedures at a freestanding neurosurgical clinic with a successful discharge rate of $99.8 \%$ on the day of surgery. ${ }^{19}$ No patients died within 30 days, and the 90-day readmission rate was $1.5 \%$. Nine patients $(0.6 \%)$ suffered a postoperative hematoma, which was recognized and evacuated postoperatively, and these patients were subsequently discharged the same day. Notably, this study was conducted in Oslo, Norway, in a health care ecosystem distinct from that of the United States. Another group of investigators studied 212 consecutive patients in the United States, who had undergone a first operation for lumbar disease; the authors reported the overall success rate at 2 years as $75 \%-$ $80 \%$, as defined by the visual analog scale and Oswestry Disability Index. ${ }^{4}$ In their cohort, the average hospital stay was 5 hours, and only 1 patient $(0.5 \%)$ was admitted to the inpatient service following surgery. Best and Sasso analyzed outcomes for 233 consecutive patients 65 years of age or older who underwent outpatient lumbar decompression, finding an inpatient admission conversion rate of $4.1 \%$ and an overall complication rate of $7.1 \% .{ }^{8}$ In addition to single-center cohort studies, the overall trends and outcomes for lumbar laminectomy and discectomy have been analyzed using large surgical databases. Pugely and colleagues performed a propensity score-matched analysis of 4310 lumbar discectomy cases in the American College of Surgeons database. ${ }^{34}$ Interestingly, in the matched cohort, the inpatient group had a significantly higher rate of complications (OR 1.521) even after adjusting for potential confounders. Moreover, an advanced age, diabetes, and operative times longer than 150 minutes were independent predictors of a postoperative complication. All data taken together, lumbar decompression has the strongest evidence for safety in the outpatient setting.

\section{Lumbar Fusion}

The literature regarding lumbar fusion in the outpatient setting is more limited than that regarding decompression with or without discectomy (Table 2). Conceptually, as minimally invasive fusion techniques continue to evolve, this is a promising group of operations to transition to outpatient procedures. Several smaller cohort studies have reported favorable outcomes from minimally invasive transforaminal lumbar interbody fusion and posterior fusion performed in the outpatient setting. ${ }^{16}$ One technical modification to posterior fusion includes the use of midline cortical bone trajectory pedicle screws to reduce the amount of muscle dissection and tissue destruction without sacrificing fusion rates. ${ }^{12}$ Another promising avenue involves the use of lateral fusion techniques, which may also reduce postoperative pain and thus enable earlier discharge. Smith et al. performed a retrospective analysis of 1033 patients treated with minimally invasive lateral interbody fusion and grouped patients according to length of stay. ${ }^{39}$ They found that a younger age, lower body mass index, less advanced disease, and higher preoperative hemoglobin levels were predictive factors for discharge within 24 hours. In the prospective arm, the authors performed 54 lateral and 18 posterior fusions in an ambulatory setting with no transfers to an inpatient facility. Two additional patients $(3.7 \%)$ visited the emergency department within 30 days. Another author group prospectively compared 70 consecutive patients undergoing lateral fusion in either an inpatient or outpatient setting. ${ }^{11}$ There were no significant baseline differences in characteristics between the two cohorts, including age, body mass index, or pathological level treated. Additionally, fusion was achieved in all patients. Between the two groups, the outpatient cohort benefited from significant improvement in the Oswestry Disability Index, less blood loss, and shorter operative time. Overall, these studies suggest that for young, healthy patients, a lateral fusion may be well tolerated with sameday discharge. However, the overall reported readmission rates tended to be higher than those in the lumbar decompression and/or discectomy literature.

\section{Anterior Cervical Discectomy and Fusion}

There is a growing body of evidence in support of anterior cervical discectomy and fusion (ACDF) performed in the outpatient setting (Table 3). However, unlike in lumbar surgery, the specter of neck hematoma and airway compromise creates an additional barrier to changes in practice.

The first reports of outpatient ACDF were small, single-surgeon feasibility studies reporting on fewer than 100 patients undergoing 1- or 2-level surgery with same- 
TABLE 1. Summary on the safety of and outcomes for outpatient lumbar laminectomy and discectomy

\begin{tabular}{|c|c|c|c|}
\hline $\begin{array}{l}\text { Authors } \\
\text { \& Year }\end{array}$ & Study Information & Type of Surgery & Observations/Conclusions \\
\hline $\begin{array}{l}\text { Asch et } \\
\text { al., } \\
2002\end{array}$ & $\begin{array}{l}\text { Single institution, prospec- } \\
\text { tive, } 212 \text { outpatients }\end{array}$ & Lumbar microdiscectomy & $\begin{array}{l}\text { Workers' comp \& age had } \\
\text { negative impact on } \\
\text { outcome }\end{array}$ \\
\hline $\begin{array}{l}\text { Best \& } \\
\text { Sasso, } \\
2007\end{array}$ & $\begin{array}{l}2 \text { surgeons, patient age } \\
\geq 65 \text { yrs, study dates } \\
1992-2001\end{array}$ & $\begin{array}{l}\text { Lumbar decompression: } \\
\qquad 1377\end{array}$ & $\begin{array}{l}\text { Lumbar spine surgical decom- } \\
\text { pression safe as outpa- } \\
\text { tient procedure in elderly } \\
\text { patients }\end{array}$ \\
\hline $\begin{array}{l}\text { Walid et } \\
\text { al., } \\
2010\end{array}$ & $\begin{array}{l}\text { Reviewed patients who went } \\
\text { through common process } \\
\text { of surgery venue selec- } \\
\text { tion: } 97 \text { outpatients, } 578 \\
\text { inpatients }\end{array}$ & $\begin{array}{l}\text { ACDF (levels unspeci- } \\
\text { fied), lumbar micro- } \\
\text { discectomy, lumbar } \\
\text { decompression w/ or } \\
\text { w/o fusion }\end{array}$ & $\begin{array}{l}\text { Mean age older in inpatients } \\
\text { ( } p<0.001) \text {; prevalence of } \\
\text { DM, CHF, heart disease, } \\
\text { CABG/stent/balloon } \\
\text { angioplasty, knee problems, } \\
\& \text { depression higher in inpa- } \\
\text { tients }(p<0.05) \text {; prevalence } \\
\text { of COPD \& history of stroke } \\
\text { higher in outpatient cervical } \\
\text { surgery cohort }(p<0.05)\end{array}$ \\
\hline $\begin{array}{l}\text { Pugely } \\
\text { et al., } \\
2013\end{array}$ & $\begin{array}{l}\text { NSQIP, study dates } \\
\text { 2005-2010, } 1652(38.3 \%) \\
\text { outpatients, } 2658(61.7 \%) \\
\text { inpatients }\end{array}$ & $\begin{array}{l}\text { Single-level lumbar disc- } \\
\text { ectomy }\end{array}$ & $\begin{array}{l}\text { Complication rate higher in } \\
\text { inpatients ( } \mathrm{p}<0.0001 \text { ); age, } \\
\text { DM, preop wound infection, } \\
\text { blood transfusion, op time, } \\
\text { \& inpatient hospital stay all } \\
\text { independent risk factors for } \\
\text { short-term complication; } \\
\text { surgeons should consider } \\
\text { outpatient surgery in ap- } \\
\text { propriate candidates }\end{array}$ \\
\hline $\begin{array}{l}\text { Lang et } \\
\quad \text { al., } \\
2014\end{array}$ & $\begin{array}{l}\text { Two academic hospitals, } \\
\text { study dates } 2008-2012, \\
368 \text { after outpatient } \\
\text { protocol, } 643 \text { before } \\
\text { outpatient protocol }\end{array}$ & Lumbar discectomy & $\begin{array}{l}\text { w/ implementation of outpa- } \\
\text { tient protocol, outpatient } \\
\text { lumbar discectomy safe \& } \\
\text { effective; improving periop } \\
\text { pain management \& ensur- } \\
\text { ing cases scheduled early } \\
\text { in the day may decrease } \\
\text { admissions }\end{array}$ \\
\hline
\end{tabular}

\begin{tabular}{|c|c|c|c|c|}
\hline & & & & (almost all dural tears) $11.9 \%$ \\
\hline $\begin{array}{l}\text { Best et al., } \\
2015\end{array}$ & $\begin{array}{l}\text { National Survey of Ambula- } \\
\text { tory Surgery }\end{array}$ & $\begin{array}{l}\text { Discectomy, laminectomy, } \\
\text { fusion }\end{array}$ & $\begin{array}{l}\text { Ambulatory surgeries for } \\
\text { intervertebral disc disorders } \\
\text { \& spinal stenosis increased } \\
\text { btwn } 1994 \text { \& } 2006\end{array}$ & \\
\hline $\begin{array}{l}\text { Helseth } \\
\text { et al., } \\
2015\end{array}$ & $\begin{array}{l}\text { Private clinic, single } \\
\text { institution, prospectively } \\
\text { recorded complications, } \\
\text { study dates } 2008-2013, \\
1449 \text { outpatients }\end{array}$ & $\begin{array}{l}\text { Microsurgical decompres- } \\
\text { sion: lumbar 1073, } \\
\text { cervical } 376\end{array}$ & $\begin{array}{l}\text { In favor of outpatient spinal } \\
\text { surgery for properly se- } \\
\text { lected patients }\end{array}$ & $\begin{array}{l}\text { Surgical mortality: } 0(0 \%) \text {, any complication: } 51 \\
(3.5 \%) \text {, same-day admission: } 3(0.2 \%) \text {, ad- } \\
\text { mission w/in } 3 \text { mos: } 22(1.5 \%) \text {, hematoma: } 9 \\
\text { (0.6\%), neurological deterioration: } 4(0.3 \%) \text {, } \\
\text { deep wound infection: } 13(0.9 \%) \text {, dural } \\
\text { lesion \& CSF leakage: } 15(1.0 \%) \text {, persistent } \\
\text { dysphagia: } 2(0.1 \%) \text {, persistent hoarseness: } \\
2(0.1 \%) \text {, severe pain/headache: } 6(0.4 \%), \\
\text { reoperation: } 67(4.6 \%) \text {, all life-threatening } \\
\text { hematomas detected w/in hrs after cervical } \\
(6 \mathrm{hrs}) \text { \& lumbar }(3 \mathrm{hrs}) \text { surgery }\end{array}$ \\
\hline
\end{tabular}


» CONTINUED FROM PAGE 3

TABLE 1. Summary on the safety of and outcomes for outpatient lumbar laminectomy and discectomy

\begin{tabular}{|c|c|c|c|}
\hline $\begin{array}{l}\text { Authors } \\
\& \text { Year }\end{array}$ & Study Information & Type of Surgery & Observations/Conclusions \\
\hline $\begin{array}{l}\text { Emami } \\
\text { et al., } \\
2016\end{array}$ & $\begin{array}{l}\text { Single institution, study } \\
\text { dates Jan-Dec 2012, } 32 \\
\text { outpatients, } 64 \text { inpatients }\end{array}$ & 1- or 2-level MI TLIFs & $\begin{array}{l}\text { Outpatients significantly } \\
\text { younger, had lower ASA } \\
\text { physical status scores \& } \\
\text { lower CCls than inpatients; } \\
\text { no statistical difference in } \\
\text { overall postop complication } \\
\text { rate, readmission rate, final } \\
\text { ODI or VAS scores }\end{array}$ \\
\hline
\end{tabular}

\begin{tabular}{|c|c|c|c|c|}
\hline $\begin{array}{l}\text { Chin et } \\
\quad \text { al., } \\
2016\end{array}$ & $\begin{array}{l}\text { Multiple institutions, } 30 \text { out- } \\
\text { patients, } 40 \text { inpatients }\end{array}$ & $\begin{array}{l}\text { Single-level LLIF w/ } \\
\text { supplemental pst } \\
\text { fixation at each lumbar } \\
\text { level from L-1 to L-5; } \\
\text { LLIF performed at } \\
\text { ASC or as inpatient } \\
\text { procedure }\end{array}$ & $\begin{array}{l}\text { LLIF as outpatient procedure } \\
\text { has significant improvement } \\
\text { in ODI scores compared to } \\
\text { scores for inpatient proce- } \\
\text { dure }(p=0.013) \text {; outpatient } \\
\text { LLIF improves patient } \\
\text { outcome } w / \text { similar safety } \\
\text { as inpatient procedure }\end{array}$ & $\begin{array}{l}\text { Complication rate for inpatient > that for ASC; } \\
\text { ASC dermatome numbness: } 2(7 \%) \text {; inpa- } \\
\text { tient dermatome numbness: } 4(10 \%) \text {; weak- } \\
\text { ness: } 3(7.5 \%) \text {; inability to walk: } 1(2.5 \%)\end{array}$ \\
\hline $\begin{array}{l}\text { Chin et } \\
\text { al., } \\
2017^{13}\end{array}$ & $\begin{array}{l}\text { Single surgeon, ASC, study } \\
\text { dates 2008-2014, } 557 \\
\text { ASC, } 210 \text { inpatients }\end{array}$ & $\begin{array}{l}\text { Inpatient: decompres- } \\
\text { sion } 71, \text { fusion } 138 ; \\
\text { outpatient: decompres- } \\
\text { sion } 150, \text { fusion/disc } \\
\text { replacement } 197\end{array}$ & $\begin{array}{l}\text { Majority of spine surgery } \\
\text { can be done as outpatient } \\
\text { procedure after meeting } \\
\text { certain eligibility criteria }\end{array}$ & $\begin{array}{l}\text { Overall revision surgery } 14 \% \text {, overall compli- } \\
\text { cation rate } 5 \%\end{array}$ \\
\hline $\begin{array}{l}\text { Idowu } \\
\text { et al., } \\
2017\end{array}$ & $\begin{array}{l}\text { Truven Health Marketscan } \\
\text { Research Databases, } \\
\text { study dates 2003-2014, } \\
\text { inpatient hospital, outpa- } \\
\text { tient hospital, ASC }\end{array}$ & $\begin{array}{l}\text { Lumbar fusion, lumbar } \\
\text { decompression, ant } \\
\text { cervical fusion, pst cer- } \\
\text { vical decompression, } \\
\text { pst cervical fusion }\end{array}$ & $\begin{array}{l}\text { True ambulatory surgeries } \\
\text { (defined as at ASC) not } \\
\text { increasing at same rate as } \\
\text { outpatient procedures }\end{array}$ & \\
\hline $\begin{array}{l}\text { Yen \& } \\
\quad \text { Albargi, } \\
2017\end{array}$ & $\begin{array}{l}\text { Single institution, } 2 \text { 18-mo } \\
\text { periods, pre- \& postimple- } \\
\text { mentation of ambulatory } \\
\text { outpatient protocol }\end{array}$ & Lumbar laminectomy & $\begin{array}{l}1 \text { readmission in inpatient co- } \\
\text { hort, outpatient \& overnight } \\
\text { laminectomy safe, out of } \\
\text { town patients also safe }\end{array}$ & $\begin{array}{l}\text { No patients required postop admission to } \\
\text { hospital or readmission in } 30 \text { days; inpatient } \\
\text { readmission: } 1\end{array}$ \\
\hline
\end{tabular}

ant = anterior; $\mathrm{ASA}=$ American Society of Anesthesiologists; $\mathrm{ASC}=$ ambulatory surgery center; $\mathrm{CABG}=$ coronary artery bypass grafting; $\mathrm{CCl}=\mathrm{Charlson} \mathrm{Comorbid}-$ ity Index; CHF = congestive heart failure; $\mathrm{COPD}=$ chronic obstructive pulmonary disease; $\mathrm{DM}=$ diabetes mellitus; $\mathrm{ED}=$ emergency department; $\mathrm{LLIF}=$ lateral lumbar interbody fusion; MI = minimally invasive; NSQIP = National Surgical Quality Improvement Program; ODI = Oswestry Disability Index; pst = posterior; SSI = surgical site infection; TLIF = transforaminal lumbar interbody fusion; VAS = visual analog scale.

day discharge..$^{38,40,42,43}$ There were no reported deaths, and overall complication rates ranged from $0 \%$ to $2 \%$. In these reports, only 1 patient required conversion to inpatient status for neck swelling and this patient did not require reoperation. The initial studies provided proof of concept but were limited by a lack of statistical power to show a difference between inpatient and outpatient ACDF. More recently, there have been several larger clinical series and database studies reporting direct comparisons of inpatient and outpatient ACDF. McGirt et al. obtained 1442 ACDF cases (650 inpatients, 792 outpatients) from the American College of Surgeons database, and after propensity matching for 32 covariates such as number of levels, medical comorbidities, age, and sex, these authors found that outpatient ACDF had $58 \%$ reduced odds of a major morbidity and $80 \%$ lower odds of reoperation within 30 days (ORs 0.42 and 0.20 , respectively) ${ }^{29}$ The same author group analyzed 1000 consecutive ACDF patients, all of whom had been observed for at least 4 hours prior to discharge. ${ }^{1}$ Notably, all of the patients had American Society of Anesthesiologists physical status class I or II, all underwent 1- or 2-level ACDF, and all cases began before noon. Overall, 8 patients $(0.08 \%)$ were transferred to inpatient status. There were no significant differences between the inpatient and outpatient cohort in the 30- and 90-day readmission or reoperation rate. Several other surgical database studies have since corroborated these findings in support of outpatient 1- or 2-level ACDF with an overall low comorbidity profile. ${ }^{17,24,35}$ Additionally, Ban and colleagues performed a meta-analysis and systematic review, 
TABLE 2. Summary on the safety of and outcomes for outpatient lumbar fusion

\begin{tabular}{|c|c|c|c|c|}
\hline $\begin{array}{l}\text { Authors } \\
\& \text { Year }\end{array}$ & Study Information & Type of Surgery & Observations/Conclusions & Outcomes \\
\hline $\begin{array}{l}\text { Walid et } \\
\text { al., } \\
2010\end{array}$ & $\begin{array}{l}\text { Reviewed patients who } \\
\text { went through common } \\
\text { process of surgery } \\
\text { venue selection: } 97 \text { out- } \\
\text { patients, } 578 \text { inpatients }\end{array}$ & $\begin{array}{l}\text { ACDF (levels unspecified), } \\
\text { lumbar microdiscectomy, } \\
\text { lumbar decompression w/ } \\
\text { or w/o fusion }\end{array}$ & $\begin{array}{l}\text { Mean age older in inpatients }(p<0.001) \text {; } \\
\text { prevalence of DM, CHF, heart disease, } \\
\text { CABG/stent/balloon angioplasty, knee } \\
\text { problems, \& depression higher in inpa- } \\
\text { tients }(p<0.05) \text {; prevalence of COPD } \\
\text { \& history of stroke higher in outpatient } \\
\text { cervical surgery cohort }(p<0.05)\end{array}$ & $\begin{array}{l}\text { Outpatients: any complication } 1 \\
(1.0 \%) \text {, postop infection } 1(1.0 \%) \text {; } \\
\text { inpatients: any complication } \\
16(2.8 \%) \text {, postop infection } 16 \\
(2.8 \%) \text {; all patients w/ complica- } \\
\text { tions obese }\end{array}$ \\
\hline $\begin{array}{l}\text { Best et } \\
\quad \text { al., } \\
2015\end{array}$ & $\begin{array}{l}\text { National Survey of Ambu- } \\
\text { latory Surgery }\end{array}$ & $\begin{array}{l}\text { Discectomy, laminectomy, } \\
\text { fusion }\end{array}$ & $\begin{array}{l}\text { Ambulatory surgeries for intervertebral } \\
\text { disc disorders \& spinal stenosis } \\
\text { increased btwn } 1994 \text { \& } 2006\end{array}$ & \\
\hline $\begin{array}{l}\text { Chin et } \\
\text { al., } \\
2017^{12}\end{array}$ & $\begin{array}{l}\text { ASC, single surgeon, } 16 \\
\text { outpatients }\end{array}$ & $\begin{array}{l}\text { 1-level PLIF or TLIF (direct } \\
\text { open, single-level PLIF) }\end{array}$ & $\begin{array}{l}\text { Direct open PLIF done safely w/ signifi- } \\
\text { cant reduction in average pain \& ODI } \\
\text { scores }\end{array}$ & $\begin{array}{l}\text { Worsened back pain \& possible } \\
\quad \text { aseptic discitis: } 1(6.3 \%)\end{array}$ \\
\hline $\begin{array}{l}\text { Chin et } \\
\quad \text { al., } \\
2016\end{array}$ & $\begin{array}{l}\text { Multiple institutions, } \\
30 \text { outpatients, } 40 \\
\text { inpatients }\end{array}$ & $\begin{array}{l}\text { Single-level LLIF w/ } \\
\text { supplemental pst fixation } \\
\text { at each lumbar level from } \\
\text { L-1 to L-5; LLIF performed } \\
\text { at ASC or as inpatient } \\
\text { procedure }\end{array}$ & $\begin{array}{l}\text { LLIF as outpatient procedure has signifi- } \\
\text { cant improvement in ODI scores com- } \\
\text { pared to scores for inpatient procedure } \\
(p=0.013) \text {; outpatient LLIF improves } \\
\text { patient outcome w/ similar safety as } \\
\text { inpatient procedure }\end{array}$ & $\begin{array}{l}\text { Complication rate for inpatients } \\
\text { > that for outpatients; ASC } \\
\text { dermatome numbness: } 2(7 \%) \text {; } \\
\text { inpatient dermatome numbness: } \\
4(10 \%) \text {, weakness: } 3(7.5 \%) \\
\text { inability to walk: } 1(2.5 \%)\end{array}$ \\
\hline $\begin{array}{c}\text { Emami } \\
\text { et al., } \\
2016\end{array}$ & $\begin{array}{l}\text { Single institution, study } \\
\text { dates Jan-Dec } 2012 \text {, } \\
32 \text { outpatients, } 64 \\
\text { inpatients }\end{array}$ & 1- or 2-level MI TLIFs & $\begin{array}{l}\text { Outpatients significantly younger, had low- } \\
\text { er ASA physical status scores \& lower } \\
\text { CCIs than inpatients; no statistical } \\
\text { difference in overall postop complica- } \\
\text { tion rate, readmission rate, final ODI or } \\
\text { VAS scores }\end{array}$ & $\begin{array}{l}\text { Outpatients: neurological 2, postop } \\
\text { hematoma } 0 \text {, incidental durotomy } \\
0, \text { SSI } 0 \text {, instrumentation (pedicle } \\
\text { screw malposition, hardware } \\
\text { prominence, rod disengagement) } \\
1 \text {; inpatients: neurological } 3 \text {, } \\
\text { postop hematoma } 2 \text {, incidental } \\
\text { durotomy } 1, \text { SSI } 3 \text {, instrumenta- } \\
\text { tion (pedicle screw malposition, } \\
\text { hardware prominence, rod } \\
\text { disengagement) } 1\end{array}$ \\
\hline $\begin{array}{l}\text { Smith } \\
\text { et al., } \\
2016\end{array}$ & $\begin{array}{l}\text { ASC, predictive arm: } 873 \\
\text { d/c }<24 \text { hrs (outpa- } \\
\text { tients), } 160 \mathrm{~d} / \mathrm{c}>23 \mathrm{hrs} \\
\text { (inpatients); clinical } \\
\text { study: } 54 \text { consecutive } \\
\text { XLIF \& } 18 \text { consecutive } \\
\text { MI pst fusion }\end{array}$ & $\begin{array}{l}\text { Lumbar fusion (1-4 levels), } \\
\text { XLIF, MI pst fusion }\end{array}$ & $\begin{array}{l}\text { Select patients can be treated as out- } \\
\text { patients w/ XLIF \& other MI surgical } \\
\text { approaches; based on predictive study: } \\
\text { younger age, higher preop hemoglo- } \\
\text { bin, fewer levels, lower BMI, \& less } \\
\text { advanced disease may predict early d/c }\end{array}$ & $\begin{array}{l}\text { Clinical study (72): no intraop or } \\
\text { postop complications in either } \\
\text { XLIF or MI pst fusion cohort; no } \\
\text { transfers to inpatient facility }\end{array}$ \\
\hline $\begin{array}{l}\text { Idowu } \\
\text { et al., } \\
2017\end{array}$ & $\begin{array}{l}\text { Truven Health Marketscan } \\
\text { Research Databases, } \\
\text { study dates 2003-2014, } \\
\text { inpatient hospital, out- } \\
\text { patient hospital, ASC }\end{array}$ & $\begin{array}{l}\text { Lumbar fusion, lumbar de- } \\
\text { compression, ant cervical } \\
\text { fusion, pst cervical de- } \\
\text { compression, pst cervical } \\
\text { fusion }\end{array}$ & $\begin{array}{l}\text { True ambulatory surgery (defined as at } \\
\text { ASC) not increasing at same rate as } \\
\text { outpatient procedures }\end{array}$ & \\
\hline $\begin{array}{l}\text { Chin et } \\
\text { al., } \\
2017^{12}\end{array}$ & $\begin{array}{l}\text { Prospective, single } \\
\text { institution, } 30 \text { CBT } \\
\text { pedicle screws OSC, } \\
30 \text { traditional pedicle } \\
\text { screws inpatient }\end{array}$ & Pst lumbar fixation & $\begin{array}{l}\text { Successful lumbar fusions in OSC using } \\
\text { midline CBT pedicle screw; traditional } \\
\text { method may still work as outpatient } \\
\text { procedure, but authors claim midline } \\
\text { technique is more advantageous; OSC } \\
\text { led to significant improvement in VAS } \\
\text { back pain ( } p=0.004 \text { ) and ODI ( } p= \\
0.027 \text { ) scores; similar fusion rate at } 2 \text { yrs }\end{array}$ & \\
\hline $\begin{array}{l}\text { Chin et } \\
\text { al., } \\
2017^{13}\end{array}$ & $\begin{array}{l}\text { Single surgeon, ASC, } \\
\text { study dates 2008-2014, } \\
557 \text { ASC, } 210 \text { inpatients }\end{array}$ & $\begin{array}{l}\text { Inpatient: decompression } 71 \text {, } \\
\text { fusion 138; outpatient: de- } \\
\text { compression 150, fusion/ } \\
\text { disc replacement } 197\end{array}$ & $\begin{array}{l}\text { Majority of spine surgery can be done } \\
\text { as outpatient procedure after meeting } \\
\text { certain eligibility criteria }\end{array}$ & $\begin{array}{l}\text { Overall revision surgery } 14 \% \text {, } \\
\text { overall complication rate } 5 \%\end{array}$ \\
\hline
\end{tabular}

$\mathrm{BMI}=$ body mass index; $\mathrm{d} / \mathrm{c}=$ discharge; LLIF or XLIF = lateral lumbar interbody fusion; CBT = cortical bone trajectory; OSC = outpatient surgery center; PLIF = posterior lumbar interbody fusion. 


\section{A. V. Pendharkar et al.}

TABLE 3. Summary on the safety of and outcomes for outpatient anterior cervical spine surgeries

\begin{tabular}{|c|c|c|c|c|}
\hline $\begin{array}{l}\text { Authors } \\
\& \text { Year }\end{array}$ & Study Information & Type of Surgery & Observations/Conclusions & Outcomes \\
\hline $\begin{array}{l}\text { Silvers } \\
\text { et al., } \\
1996\end{array}$ & $\begin{array}{l}\text { Single institution, study } \\
\text { dates May-Dec } 1994, \\
50 \text { prospectively } \\
\text { analyzed outpatients, } \\
53 \text { retrospectively ana- } \\
\text { lyzed inpatient controls }\end{array}$ & 1- to 2-level ACDF & $\begin{array}{l}\text { No statistically significant difference btwn } \\
\text { inpatient \& outpatient groups on any } \\
\text { parameters; ambulatory surgery does } \\
\text { not compromise safety or efficacy of } \\
\text { ACDF }\end{array}$ & $\begin{array}{l}\text { Mortality: } 0 \% \text {, complication rate for each } \\
\text { group: } 2 \% \text {; outpatients: dysphagia (par- } \\
\text { tially recovered) \& vocal cord paralysis } \\
\text { (not fully resolved at }>1 \text { yr): } 1(2 \%) ; \\
\text { inpatients: superficial wound infection: } \\
1(1.9 \%)\end{array}$ \\
\hline $\begin{array}{c}\text { Trahan } \\
\text { et al., } \\
2011\end{array}$ & $\begin{array}{l}\text { One physician's practice, } \\
\text { study dates Nov } 2005- \\
\text { Apr } 2009,59 \text { outpa- } \\
\text { tients, } 58 \text { inpatients }\end{array}$ & $\begin{array}{l}\text { 1- to 2-level ACDF: } \\
\text { 1-level 68, 2-level } \\
49\end{array}$ & $\begin{array}{l}\text { 1- to 2-level ACDF can be done on an } \\
\text { outpatient basis; complication rates } \\
\text { low, critical postop complications in- } \\
\text { cluding respiratory compromise occur } \\
\text { very infrequently \& in the immediate } \\
\text { postop period }\end{array}$ & $\begin{array}{l}\text { Outpatients: any complication } 1(1.4 \%) \\
\text { neck swelling \& difficulty breathing \& } \\
\text { anxiety requiring readmission } 1(1.4 \%)\end{array}$ \\
\hline $\begin{array}{r}\text { Stieber } \\
\text { et al., } \\
2005\end{array}$ & $\begin{array}{l}\text { Two senior authors, free- } \\
\text { standing ASC, study } \\
\text { dates } 1998-2002,30 \\
\text { ASC, } 60 \text { inpatients }\end{array}$ & $\begin{array}{l}\text { 1- to 2-level ACDF+P } \\
\text { at C4-5 or below } \\
\text { as adjunct to } \\
\text { autogenous iliac } \\
\text { crest bone graft } \\
\text { or structural al- } \\
\text { lograft: } 1 \text {-level: } 40 \text {, } \\
\text { 2-level: } 50\end{array}$ & $\begin{array}{l}\text { Outpatient group had lower complication } \\
\text { rate than controls (likely due to selec- } \\
\text { tion bias); transient dysphagia most } \\
\text { common complication in outpatients }\end{array}$ & $\begin{array}{l}\text { ASC: any complication } 3(10 \%) \text {, dysphagia } \\
3(10 \%) \text {, readmission } 0(0 \%) \text {; inpatients: } \\
\text { any complication } 7(13 \%) \text {, transient } \\
\text { dysphagia } 3(5 \%) \text {, graft donor site pain } 4 \\
(14 \%) \text {, increased LOS due to com- } \\
\text { plication } 4(7 \%) \text {, readmitted for early } \\
\text { complication } 4(7 \%)\end{array}$ \\
\hline $\begin{array}{l}\text { Lied et } \\
\quad \text { al., } \\
2008\end{array}$ & $\begin{array}{l}\text { Single institution, } 390 \\
\text { outpatients }\end{array}$ & $\begin{array}{l}\text { ACDF: } 278 \text { fused w/ } \\
\text { autologous iliac } \\
\text { crest, } 112 \text { fused w/ } \\
\text { PEEK graft }\end{array}$ & $\begin{array}{l}\text { 6-hr postop observation, then discharge } \\
\text { is safe }\end{array}$ & $\begin{array}{l}\text { Mortality: } 0 \text { (0\%); any complication: } 37 \\
\text { (9\%), immediate complication (0-6 hrs): } \\
\text { 17, early complication (6-72 hrs): } 1 \text {, } \\
\text { late complication (>72 hrs): 19; all life- } \\
\text { threatening neck hematomas detected } \\
\text { w/in first } 6 \text { hrs }\end{array}$ \\
\hline $\begin{array}{l}\text { Villavi- } \\
\text { cencio } \\
\text { et al., } \\
2007\end{array}$ & $\begin{array}{l}\text { Single institution, study } \\
\text { dates Apr 2003-Apr } \\
\text { 2005, } 103 \text { outpatients, } \\
\text { d/c <15 hrs postop: } 99 \\
\text { (96.1\%), d/c after } 23 \\
\text { hrs observation after } \\
\text { 3-level ACDF: } 4(3.9 \%)\end{array}$ & 1- to 3-level ACDF & $\begin{array}{l}\text { ACDF w/ instrumentation as outpatient } \\
\text { is safe \& feasible \& not associated w/ } \\
\text { increased complications }\end{array}$ & $\begin{array}{l}\text { Overall complication rate: } 4 \text { (3.8\%), major } \\
\text { complications (vertebral fracture \& } \\
\text { dehydration resulting in readmission): } \\
2(1.9 \%) \text {, minor complications (allergic } \\
\text { reaction to medications that did not re- } \\
\text { quire hospitalization, transient [ } \leq 3 \text { mos] } \\
\text { neurological deficit): } 2(1.9 \%)\end{array}$ \\
\hline $\begin{array}{l}\text { Garringer } \\
\quad \& \\
\text { Sasso, } \\
2010\end{array}$ & $\begin{array}{l}\text { Single surgeon, prospec- } \\
\text { tive, study dates Nov } \\
\text { 1993-May 2006, } 645 \\
\text { outpatients }\end{array}$ & 1-level ACDF & $\begin{array}{l}\text { 1-level ACDF safe in outpatient setting w/ } \\
\text { 4-hr observation; using postop drain is } \\
\text { questionable }\end{array}$ & $\begin{array}{l}\text { Mortality: } 0(0 \%) \text {, any complication: } 2 \\
(0.3 \%), \text { both epidural hematomas, both } \\
\text { occurred w/in } 4 \text {-hr observation period, } \\
\text { both resolved w/o permanent deficit; } \\
\text { unplanned admission: } 24(6 \%),>80 \% \\
\text { due to pain or nausea }\end{array}$ \\
\hline $\begin{array}{l}\text { Sheperd } \\
\text { \& } \\
\text { Young, } \\
2012\end{array}$ & $\begin{array}{l}\text { ASC dedicated to spine } \\
\text { surgery, study dates } \\
\text { 2007-2009, 152 ASC }\end{array}$ & 1- to 2-level ACDF & $\begin{array}{l}75 \text { patients completed self-reported } \\
\text { survey w/in } 6 \text { mos, reporting } 100 \% \text { sat- } \\
\text { isfaction rate; ACDF safe in selected } \\
\text { patients as outpatient procedure w/ } \\
\text { high patient satisfaction }\end{array}$ & $\begin{array}{l}\text { ED visit } 6(3.9 \%) \text { : neck pain } 2(1.3 \%) \text {, } \\
\text { dysphagia } 1(0.7 \%) \text {, vocal cord paralysis } \\
\text { \& dysphagia } 1(0.7 \%) \text {, nausea } 1(0.7 \%) \text {, } \\
\text { cervical swelling } 1(0.7 \%) \text {; required } \\
\text { readmission: } 1(0.7 \%) \text {; long-term se- } \\
\text { quelae: } 0(0 \%) \text {; complication rate: } 3.9 \%\end{array}$ \\
\hline $\begin{array}{r}\text { Wohns, } \\
2010\end{array}$ & $\begin{array}{l}\text { Single institution, study } \\
\text { dates Feb 2009-May } \\
\text { 2010, } 14 \text { ASC, } 12 \\
\text { hospital-based outpa- } \\
\text { tients }\end{array}$ & $\begin{array}{l}\text { Cervical disc arthro- } \\
\text { plasty }\end{array}$ & $\begin{array}{l}\text { 100\% patients reported improvement; } \\
\text { outpatient cervical disc arthroplasty } \\
\text { costs: } 62 \%<1 \text {-level outpatient ACDF, } \\
84 \%<1 \text {-level inpatient cervical disc ar- } \\
\text { throplasty; outpatient: } 1 \text {-level cervical } \\
\text { disc arthroplasty: } \$ 11,144.83,1 \text {-level } \\
\text { ACDF: } \$ 29,313.43 ; \text { inpatient: 1-level } \\
\text { cervical disc arthroplasty: } \$ 68,000, \\
\text { 1-level ACDF: } \$ 61,095.49\end{array}$ & $\begin{array}{l}\text { No mortality, complications, cases requir- } \\
\text { ing hospital transfer, postop ED visit }\end{array}$ \\
\hline
\end{tabular}


» CONTINUED FROM PAGE 6

TABLE 3. Summary on the safety of and outcomes for outpatient anterior cervical spine surgeries

\begin{tabular}{|c|c|c|c|c|}
\hline $\begin{array}{l}\text { Authors } \\
\& \text { Year }\end{array}$ & Study Information & Type of Surgery & Observations/Conclusions & Outcomes \\
\hline $\begin{array}{l}\text { Walid et } \\
\text { al., } \\
2010\end{array}$ & $\begin{array}{l}\text { Reviewed patients who } \\
\text { went through common } \\
\text { process of surgery } \\
\text { venue selection, } \\
97 \text { outpatients, } 578 \\
\text { inpatients }\end{array}$ & $\begin{array}{l}\text { ACDF (levels un- } \\
\text { specified), lumbar } \\
\text { microdiscectomy, } \\
\text { lumbar decom- } \\
\text { pression w/ or w/o } \\
\text { fusion }\end{array}$ & $\begin{array}{l}\text { Mean age older in inpatients }(p<0.001) \text {; } \\
\text { prevalence of DM, CHF, heart disease, } \\
\text { CABG/stent/balloon angioplasty, knee } \\
\text { problems, \& depression higher in inpa- } \\
\text { tients }(p<0.05) \text {; prevalence of COPD } \\
\text { \& history of stroke higher in outpatient } \\
\text { cervical surgery cohort }(p<0.05)\end{array}$ & $\begin{array}{l}\text { Outpatients: any complication } 1(1.0 \%) \text {, } \\
\text { postop infection } 1(1.0 \%) \text {; inpatients: any } \\
\text { complication } 16(2.8 \%) \text {, postop infection } \\
16(2.8 \%) ; \text { all patients w/ complications } \\
\text { obese }\end{array}$ \\
\hline $\begin{array}{l}\text { Lied et } \\
\text { al., } \\
2013\end{array}$ & $\begin{array}{l}\text { Single institution, } 96 \\
\text { outpatients }\end{array}$ & $\begin{array}{l}\text { 1- or 2-level ACDF: } \\
\text { 1-level: } 60 \\
\text { 2-level: } 36\end{array}$ & $\begin{array}{l}\text { 91\% patient satisfaction using NASSQ; } \\
\text { ACDF in select patients w/ cervical } \\
\text { disc degeneration appears safe as } \\
\text { outpatient procedure w/ sufficient } \\
\text { postop observation; clinical outcomes } \\
\text { \& patient satisfaction comparable w/ } \\
\text { those for inpatient procedure }\end{array}$ & $\begin{array}{l}\text { Mortality: } 0 \% \text {; surgical morbidity: } 5.2 \% \text {, } \\
\text { hematoma } 2(2.1 \%) \text {, dysphagia } 2(2.1 \%) \text {, } \\
\text { neurological deterioration } 1(1 \%)\end{array}$ \\
\hline $\begin{array}{l}\text { Baird et } \\
\text { al., } \\
2014\end{array}$ & $\begin{array}{l}\text { US HCUP SID \& SASD } \\
\text { for CA, NY, FL, \& MD; } \\
\text { study dates 2005-2009 }\end{array}$ & $\begin{array}{l}\text { Cervical spine sur- } \\
\text { gery in outpatient } \\
\text { setting }\end{array}$ & $\begin{array}{l}\text { Increase in cervical spine surgeries in } \\
\text { ambulatory setting during study period: } \\
\text { ACDF } 68 \% \text {, pst decompression } 21 \% \text {; } \\
\text { majority (>99\%) d/c home after ambu- } \\
\text { latory surgery }\end{array}$ & \\
\hline $\begin{array}{l}\text { Martin } \\
\text { et al., } \\
2008\end{array}$ & $\begin{array}{l}\text { NSQIP, } 597 \text { outpatients, } \\
2317 \text { inpatients }\end{array}$ & 1-level ACDF & $\begin{array}{l}\text { Age }>65 \text { yrs, ASA score III or IV, current } \\
\text { dialysis, current steroid use, recent } \\
\text { sepsis, \& op times }>120 \text { mins all inde- } \\
\text { pendent risk factors for complications; } \\
\text { no significant differences in complica- } \\
\text { tion rate btwn groups; reasonable to } \\
\text { consider inpatient 1-level ACDF in } \\
\text { patients w/ aforementioned risk factors }\end{array}$ & $\begin{array}{l}\text { Mortality: } 5(0.2 \%) \text {, any complication: } \\
92(3.2 \%) \text {, reoperation: } 34(1.2 \%) ; \\
\text { outpatients: mortality } 1(0.2 \%) \text {, any } \\
\text { complication }(1.3 \%) \text {, reoperation }(0.2 \%) \text {; } \\
\text { inpatients: mortality } 4(0.2 \%) \text {, any com- } \\
\text { plication }(3.6 \%) \text {, reoperation }(1.4 \%)\end{array}$ \\
\hline $\begin{array}{l}\text { Best et } \\
\text { al., } \\
2015\end{array}$ & $\begin{array}{l}\text { National Survey of Ambu- } \\
\text { latory Surgery }\end{array}$ & $\begin{array}{l}\text { Discectomy, laminec- } \\
\text { tomy, fusion }\end{array}$ & $\begin{array}{l}\text { Ambulatory surgeries for intervertebral } \\
\text { disc disorders \& spinal stenosis } \\
\text { increased btwn } 1994 \text { \& } 2006\end{array}$ & \\
\hline $\begin{array}{l}\text { Helseth } \\
\text { et al., } \\
2015\end{array}$ & $\begin{array}{l}\text { Private clinic, single in- } \\
\text { stitution, prospectively } \\
\text { recorded complica- } \\
\text { tions, study dates } \\
2008-2013,1449 \\
\text { outpatients }\end{array}$ & $\begin{array}{l}\text { Microsurgical } \\
\text { decompression: } \\
\text { lumbar } 1073 \text {, } \\
\text { cervical } 376\end{array}$ & $\begin{array}{l}\text { In favor of outpatient spinal surgery for } \\
\text { properly selected patients }\end{array}$ & $\begin{array}{l}\text { Surgical mortality: } 0(0 \%) \text {, any complica- } \\
\text { tion: } 51(3.5 \%) \text {, same-day admission: } \\
3(0.2 \%) \text {, admission w/in } 3 \text { mos: } 22 \\
(1.5 \%) \text {, hematoma: } 9(0.6 \%) \text {, neurologi- } \\
\text { cal deterioration: } 4(0.3 \%) \text {, deep wound } \\
\text { infection } 13(0.9 \%) \text {, dural lesion \& CSF } \\
\text { leakage: } 15(1.0 \%) \text {, persistent dyspha- } \\
\text { gia: } 2(0.1 \%), \text { persistent hoarseness: } 2 \\
\text { (0.1\%), severe pain/headache: } 6(0.4 \%) \text {, } \\
\text { reoperation: } 67(4.6 \%) \text {; all life-threaten- } \\
\text { ing hematomas detected w/in hrs after } \\
\text { cervical (6 hrs) \& lumbar ( } 3 \text { hrs) surgery }\end{array}$ \\
\hline $\begin{array}{l}\text { McGirt } \\
\text { et al., } \\
2015\end{array}$ & $\begin{array}{l}\text { NSQIP, study dates } 2005- \\
2011,1168 \text { outpatients, } \\
6120 \text { inpatients }\end{array}$ & 1- to 2-level ACDF & $\begin{array}{l}\text { Return to OR w/in } 30 \text { days \& major mor- } \\
\text { bidity lower in outpatients }\end{array}$ & $\begin{array}{l}\text { Outpatients: major morbidity } 0.94 \% \text {, return } \\
\text { to OR w/in } 30 \text { days } 1.4 \% \text {; inpatients: } \\
\text { major morbidity } 4.5 \% \text {, return to OR w/in } \\
30 \text { days } 2 \%\end{array}$ \\
\hline $\begin{array}{l}\text { Adamson } \\
\text { et al., } \\
2016\end{array}$ & $\begin{array}{l}\text { Single institution, ASC, } \\
\text { study dates 2006- } \\
2013,1000 \text { ASC, } 484 \\
\text { inpatients }\end{array}$ & $\begin{array}{l}\text { 1-, 2-, >2-level ACDF; } \\
\text { ASC: 1-level } \\
\text { 629, 2-level 365, } \\
\text { >2-level 6; inpa- } \\
\text { tient: } 1 \text {-level 274, } \\
\text { 2-level } 210\end{array}$ & $\begin{array}{l}\text { Surgical complications low \& can be } \\
\text { diagnosed in 4-hr ASC PACU window; } \\
\text { similar results compared to those for } \\
\text { inpatient ACDF; can perform ACDF } \\
\text { safely as outpatient ASC procedure; } \\
\text { 90-day morbidity similar btwn cohorts } \\
\text { for } 1 \text { - \& 2-level ACDF }\end{array}$ & $\begin{array}{l}\text { Transfer from ASC to inpatient: } 8(0.8 \%) \text {, } \\
\text { pain control: } 3 \text {, chest pain \& EEG } \\
\text { changes: } 2 \text {, intraop CSF leak: } 1 \text {, } \\
\text { postop hematoma: } 1 \text {, profound postop } \\
\text { weakness \& surgical re-exploration: } 1 \text {; } \\
\text { mortality: } 0 \% \text {; } 30 \text {-day hospital readmis- } \\
\text { sion: } 2.2 \%\end{array}$ \\
\hline
\end{tabular}




\section{A. V. Pendharkar et al.}

» CONTINUED FROM PAGE 7

TABLE 3. Summary on the safety of and outcomes for outpatient anterior cervical spine surgeries

\begin{tabular}{|c|c|c|c|c|}
\hline $\begin{array}{l}\text { Authors } \\
\text { \& Year }\end{array}$ & Study Information & Type of Surgery & Observations/Conclusions & Outcomes \\
\hline $\begin{array}{l}\text { Arshi et } \\
\text { al., } \\
2017\end{array}$ & $\begin{array}{l}\text { Humana-insured patients, } \\
\text { study dates } 2011-2016 \text {, } \\
1215 \text { outpatients, } \\
\text { 10,964 inpatient }\end{array}$ & 1- to 2-level ACDF & $\begin{array}{l}\text { Adjusting for age, sex, \& comorbidities: } \\
\text { outpatients more likely to undergo revi- } \\
\text { sion surgery for pst fusion at } 6 \text { mos \& } \\
1 \text { yr, ant fusion at } 1 \mathrm{yr} \text {; outpatient more } \\
\text { likely to have postop acute renal failure }\end{array}$ & $\begin{array}{l}\text { Outpatients: acute renal failure } 15(1.23 \%) \text {, } \\
\text { respiratory failure } 16(1.32 \%), \text { CVA } 12 \\
(0.99 \%) \text {; inpatients: acute renal failure } \\
164(1.50 \%) \text {, respiratory failure } 313 \\
(2.85 \%), \text { CVA } 132(1.20)\end{array}$ \\
\hline $\begin{array}{l}\text { Chin et } \\
\text { al., } \\
2017^{14}\end{array}$ & Single center, ASC & $\begin{array}{l}\text { TDR: } 55 ; 1 \text {-level } \\
\text { ACDF: } 55\end{array}$ & $\begin{array}{l}\text { 1-level TDR safe in ASC w/ satisfactory } \\
\text { clinical \& patient-reported outcomes; } \\
\text { comparable w/ ACDF in outpatient } \\
\text { setting }\end{array}$ & $\begin{array}{l}\text { Dysphagia most common postop complaint } \\
\text { in both groups ( } 6 \text { total), no intergroup } \\
\text { significant differences }\end{array}$ \\
\hline $\begin{array}{l}\text { Chin et } \\
\text { al., } \\
2017^{13}\end{array}$ & $\begin{array}{l}\text { Single surgeon, ASC, } \\
\text { study dates 2008- } \\
2014,557 \text { ASC, } 210 \\
\text { inpatients }\end{array}$ & $\begin{array}{l}\text { Inpatient: decom- } \\
\text { pression } 71, \text { fusion } \\
\text { 138; outpatient: } \\
\text { decompression } \\
\text { 150, fusion/disc } \\
\text { replacement } 197\end{array}$ & $\begin{array}{l}\text { Majority of spine surgery can be done } \\
\text { as outpatient procedure after meeting } \\
\text { certain eligibility criteria }\end{array}$ & $\begin{array}{l}\text { Overall revision surgery } 14 \% \text {, overall } \\
\text { complication rate } 5 \%\end{array}$ \\
\hline $\begin{array}{l}\text { Idowu } \\
\text { et al., } \\
2017\end{array}$ & $\begin{array}{l}\text { Truven Health Marketscan } \\
\text { Research Databases, } \\
\text { study dates 2003- } \\
\text { 2014, inpatient hospital, } \\
\text { outpatient hospital, } \\
\text { ASC }\end{array}$ & $\begin{array}{l}\text { Lumbar fusion, lum- } \\
\text { bar decompres- } \\
\text { sion, ant cervical } \\
\text { fusion, pst cervical } \\
\text { decompression, } \\
\text { pst cervical fusion }\end{array}$ & $\begin{array}{l}\text { True ambulatory surgery (defined as at } \\
\text { ASC) not increasing at same rate as } \\
\text { outpatient procedures }\end{array}$ & \\
\hline $\begin{array}{c}\text { Fu et al., } \\
2017\end{array}$ & $\begin{array}{l}\text { NSQIP database, study } \\
\text { dates 2011-2014, } 4759 \\
\text { outpatients, } 17,211 \\
\text { inpatients }\end{array}$ & $\begin{array}{l}\text { 1- to 2-level ACDF: } \\
\text { 2-level } 6890 \\
\text { (20.7\% outpatient) }\end{array}$ & $\begin{array}{l}\text { Greater comorbidity burden (CCI), higher } \\
\text { ASA class, chronic steroid use, HTN, } \\
\text { \& male sex independent risk factors } \\
\text { for post-d/c complications; outpa- } \\
\text { tient 2-level ACDF not associated w/ } \\
\text { increased postop morbidity relative to } \\
\text { inpatient procedure }\end{array}$ & $\begin{array}{l}\text { 2-level ACDF complications: } 1.47 \% \text { outpa- } \\
\text { tient, } 3.94 \% \text { inpatient }(p<0.001)\end{array}$ \\
\hline $\begin{array}{c}\text { Khanna } \\
\text { et al., } \\
2018\end{array}$ & $\begin{array}{l}\text { NSQIP, study dates } \\
\text { 2011-2013, } 1778 \\
\text { (25.6\%) outpatients, } \\
5162(74.4 \%) \text { inpatients }\end{array}$ & 1-level ACDF 6940 & $\begin{array}{l}\text { Complication rate higher in inpatient } \\
\text { group ( } p=0.003 \text { ); outpatient surgery } \\
\text { for } 1 \text {-level ACDF safe \& favorable for } \\
\text { select patients }\end{array}$ & $\begin{array}{l}\text { Overall complication rate: } 4.2 \% \text {; outpatient: } \\
\text { complication rate } 1.2 \%, 30 \text {-day read- } \\
\text { mission } 1.8 \% \text {, mortality } 0.1 \% \text {; inpatient: } \\
\text { complication rate } 2.5 \%, 30 \text {-day read- } \\
\text { mission } 2.2 \% \text {, mortality } 0.1 \%\end{array}$ \\
\hline $\begin{array}{l}\text { Purger } \\
\text { et al., } \\
2017\end{array}$ & $\begin{array}{l}\text { CA, FL, NY SID \& SASD, } \\
3135 \text { ambulatory, } \\
46,966 \text { inpatients }\end{array}$ & ACDF & $\begin{array}{l}\text { Ambulatory younger (48.0 vs } 53.1 \\
\text { yrs), more likely white; higher CCI, } \\
\text { increased rate of ED visits, \& readmis- } \\
\text { sion in both groups; overall charges } \\
\text { lower for ambulatory } \$ 33,362.51 \text { vs } \\
\text { inpatient } \$ 74,667.04\end{array}$ & $\begin{array}{l}\text { Ambulatory: mortality 0\%, ED w/in } 30 \\
\text { days } 168(5.4 \%), \text { readmitted } 51(1.6 \%) \text {, } \\
\text { reoperation } 200(0.4 \%) \text {; infection, } \\
\text { hematoma, disruption of surgical site } \\
\text { or complication from implant: } 20, \text { neck } \\
\text { pain or injury, radiculopathy, DD: } 52 \text {, } \\
\text { laryngeal/airway: } 0 \text {, dysphagia/esopha- } \\
\text { geal: } 7, \text { other: } 172 ; \text { inpatient: infection, } \\
\text { hematoma, disruption of surgical site } \\
\text { or complication from implant: } 397, \text { neck } \\
\text { pain or injury, radiculopathy, DD: } 630, \\
\text { laryngeal/airway: } 7, \text { dysphagia/esopha- } \\
\text { geal: } 118, \text { other: } 3792\end{array}$ \\
\hline
\end{tabular}

ACDF+P = ACDF with plating; CVA = cerebrovascular accident; $D D=$ degenerative disease; $E E G$ = electroencephalography; HCUP = United States Healthcare and Cost Utilization Project; HTN = hypertension; LOS = length of stay; NASSQ = North American Spine Society Questionnaire; OR = operating room; PACU = post anesthesia care unit; PEEK = polyetheretherketone; SID = State Inpatient Databases; SASD = State Ambulatory Surgery and Services Databases; TDR = total disc replacement. 
including 12 articles and 1693 treated levels, which revealed an overall complication rate of $1.71 \%$ and a risk ratio of 0.99 , suggesting no statistical difference between inpatient and outpatient groups. ${ }^{6}$

There may be a longer-term negative effect of outpatient ACDF. Arshi et al. examined more than 12,000 patients in a private insurance database and reported that outpatient ACDF was associated with higher odds of repeat anterior surgery at 1 year (OR 1.46) as well as a higher likelihood of undergoing posterior surgery at 6 months and 1 year (ORs 1.58 and 1.79, respectively). ${ }^{3}$ The authors speculate that pressures for high throughput in an ambulatory setting may force surgeons to be less rigorous in endplate preparation, discectomy, or proper instrumentation, leading to higher rates of pseudarthrosis. Another interesting theory posits that the bias against the treatment of more than 2 levels may increase the proportion of patients with untreated milder adjacent segment disease, which subsequently progresses. Their findings underline the importance of studying longer-term outcomes beyond 30 or 90 days to truly evaluate whether outpatient spine surgery has an unanticipated impact.

\section{Cervical Disc Arthroplasty}

Cervical disc arthroplasty is a logical companion to outpatient ACDF and may actually lend itself to superior outcomes as patients in these cases are often younger with fewer baseline comorbidities. Moreover, the surgical principles favor less bony and soft tissue disruption. For now, the data on outpatient surgery are limited. Wohns reported on a personal series of 26 consecutive cervical disc arthroplasties with a minimum 4-hour observation period in a cohort of patients with a mean age of 46 years and no comorbidities. ${ }^{46}$ There were no transfers to inpatient status, nor any readmissions or reoperations within 30 days. Another group compared 55 outpatient disc arthroplasty cases to an outpatient ACDF control group (55 patients) and again found no readmissions or reoperations within 30 days. ${ }^{14}$

\section{Cost}

As described earlier, the difference between outpatient surgery performed at a hospital and that performed at an ambulatory center confounds direct comparison of the cost savings. However, in single-center studies, several authors have reported their own cost savings. For example, performing lumbar laminectomy in an ambulatory surgery center can produce a $30 \%$ facility fee reduction. ${ }^{29}$ Similarly, Silvers et al. reported a cost savings of $\$ 1800$ per ACDF performed in 1996 and estimated a cost savings of \$140 million nationwide for that same year if every 1- or 2-level ACDF were performed in the outpatient setting. ${ }^{38}$ Wohns found the cost of a single-level outpatient cervical disc arthroplasty to be $62 \%$ less than an outpatient ACDF and $84 \%$ less than an inpatient cervical disc arthroplasty. ${ }^{46}$ This suggests that cost is a complex result of procedure, instrumentation, facility fee, and length of stay. ${ }^{30}$ Purger et al. modeled costs and charges including all complications, readmissions, and reoperations within 90 days as a bundled charge and found significant savings in the outpatient ACDF cohort-nearly half the total for inpatient ACDF. ${ }^{35}$
The 90-day bundled charge represents one of the proposed Medicare value-based reimbursement paradigms and is an ideal metric for future cost studies.

\section{Patient Selection and Discharge Criteria}

If the outcomes of ambulatory spine surgery are deemed acceptable, the next critical step will be to create protocols and standardize patient selection and postoperative care. As seen in the previously described outcome studies, there is an inherent selection bias toward younger and healthier patients undergoing outpatient spine surgery. ${ }^{44}$ Age alone has been shown to be an independent risk factor for 30day complications after $\mathrm{ACDF}^{9}$ Chin et al. analyzed the overall eligibility of patients meeting predetermined outpatient criteria in their practice, including a body mass index less than 42, a low to moderate surgical risk, and the absence of medical comorbidities. ${ }^{13}$ Interestingly, they did not include patient age but added local caregiver and close to the hospital in their protocol. Overall, in their private practice group, $79 \%$ of patients met these criteria. Along the same lines, multiple groups have discussed the need for discharge criteria. Outpatient ACDF carries the feared complication of delayed neck hematoma, and there may be an optimal postoperative observation period to prevent any delayed complications. Lied et al. studied the timing in detecting a postoperative complication after ACDF. ${ }^{27}$ Thirty-seven patients (9\%) among 390 consecutive surgeries experienced any surgical complication. When stratified by the timing of presentation-immediate (within 6 hours), early (6-72 hours), and late (greater than 72 hours) -all 5 patients $(1.2 \%)$ who developed a neck hematoma had been diagnosed and undergone evacuation within 6 hours.

Similarly, several groups have created protocols and discharge criteria for outpatient surgery..$^{15,18,25,31}$ This includes the empowerment of anesthesia colleagues and nursing staff to improve efficiency and implement safety checkpoints. ${ }^{41}$ Furthermore, the utilization of a next-day clinic visit or follow-up telephone call can maintain patient satisfaction as well as preserve safety and outcomes..$^{20,26,37}$

\section{Surgeon Preference}

One additional consideration highlights the role of surgeon preference. In the United States medicolegal environment, the impact of a single death cannot be understated from the perspective of cost as well as surgeon willingness to send a patient home early. ${ }^{45}$ For ACDF and cervical disc arthroplasty specifically, this may prevent the adoption of outpatient surgery at large regardless of the outcomes.

\section{Conclusions}

As the economic burden of United States health care continues to increase, we are obligated to produce novel solutions to rising costs. Here, we present evidence describing ambulatory spine surgery outcomes with related proposed cost savings. With proper patient selection and close follow-up, outpatient surgery may be an ideal model for innovation and significant cost reduction.

\section{References}

1. Adamson T, Godil SS, Mehrlich M, Mendenhall S, Asher 
AL, McGirt MJ: Anterior cervical discectomy and fusion in the outpatient ambulatory surgery setting compared with the inpatient hospital setting: analysis of 1000 consecutive cases. J Neurosurg Spine 24:878-884, 2016

2. Ahn J, Bohl DD, Tabaraee E, Basques BA, Singh K: Current trends in outpatient spine surgery. Clin Spine Surg 29:384386, 2016

3. Arshi A, Wang C, Park HY, Blumstein GW, Buser Z, Wang JC, et al: Ambulatory anterior cervical discectomy and fusion is associated with a higher risk of revision surgery and perioperative complications: an analysis of a large nationwide database. Spine J [epub ahead of print], 2017

4. Asch HL, Lewis PJ, Moreland DB, Egnatchik JG, Yu YJ, Clabeaux DE, et al: Prospective multiple outcomes study of outpatient lumbar microdiscectomy: should 75 to $80 \%$ success rates be the norm? J Neurosurg 96 (1 Suppl):34-44, 2002

5. Baird EO, Egorova NN, McAnany SJ, Qureshi SA, Hecht AC, Cho SK: National trends in outpatient surgical treatment of degenerative cervical spine disease. Global Spine J 4:143-150, 2014

6. Ban D, Liu Y, Cao T, Feng S: Safety of outpatient anterior cervical discectomy and fusion: a systematic review and meta-analysis. Eur J Med Res 21:34, 2016

7. Best MJ, Buller LT, Eismont FJ: National trends in ambulatory surgery for intervertebral disc disorders and spinal stenosis: a 12-year analysis of the national surveys of ambulatory surgery. Spine (Phila Pa 1976) 40:1703-1711, 2015

8. Best NM, Sasso RC: Outpatient lumbar spine decompression in 233 patients 65 years of age or older. Spine (Phila Pa 1976) 32:1135-1140, 2007

9. Buerba RA, Giles E, Webb ML, Fu MC, Gvozdyev B, Grauer $\mathrm{JN}$ : Increased risk of complications after anterior cervical discectomy and fusion in the elderly: an analysis of 6253 patients in the American College of Surgeons National Surgical Quality Improvement Program database. Spine (Phila Pa 1976) 39:2062-2069, 2014

10. Cenic A, Kachur E: Lumbar discectomy: a national survey of neurosurgeons and literature review. Can J Neurol Sci 36:196-200, 2009

11. Chin KR, Pencle FJR, Coombs AV, Brown MD, Conklin KJ, O’Neill AM, et al: Lateral lumbar interbody fusion in ambulatory surgery centers: patient selection and outcome measures compared with an inhospital cohort. Spine (Phila Pa 1976) 41:686-692, 2016

12. Chin KR, Pencle FJR, Coombs AV, Elsharkawy M, Packer $\mathrm{CF}$, Hothem EA, et al: Clinical outcomes with midline cortical bone trajectory pedicle screws versus traditional pedicle screws in moving lumbar fusions from hospitals to outpatient surgery centers. Clin Spine Surg 30:E791-E797, 2017

13. Chin KR, Pencle FJR, Coombs AV, Packer CF, Hothem EA, Seale JA: Eligibility of outpatient spine surgery candidates in a single private practice. Clin Spine Surg 30:E1352-E1358, 2017

14. Chin KR, Pencle FJR, Seale JA, Pencle FK: Clinical outcomes of outpatient cervical total disc replacement compared with outpatient anterior cervical discectomy and fusion. Spine (Phila Pa 1976) 42:E567-E574, 2017

15. Debono B, Sabatier P, Garnault V, Hamel O, Bousquet P, Lescure JP, et al: Outpatient lumbar microdiscectomy in France: from an economic imperative to a clinical standard-an observational study of 201 cases. World Neurosurg 106:891-897, 2017

16. Emami A, Faloon M, Issa K, Shafa E, Pourtaheri S, Sinha K, et al: Minimally invasive transforaminal lumbar interbody fusion in the outpatient setting. Orthopedics 39:e1218e1222, 2016

17. Fu MC, Gruskay JA, Samuel AM, Sheha ED, Derman PB, Iyer S, et al: Outpatient anterior cervical discectomy and fusion is associated with fewer short-term complications in one- and two-level cases: a propensity-adjusted analysis. Spine (Phila Pa 1976) 42:1044-1049, 2017

18. Garringer SM, Sasso RC: Safety of anterior cervical discectomy and fusion performed as outpatient surgery. J Spinal Disord Tech 23:439-443, 2010

19. Helseth $\varnothing$, Lied B, Halvorsen CM, Ekseth K, Helseth E: Outpatient cervical and lumbar spine surgery is feasible and safe: a consecutive single center series of 1449 patients. Neurosurgery 76:728-738, 2015

20. Hersht M, Massicotte EM, Bernstein M: Patient satisfaction with outpatient lumbar microsurgical discectomy: a qualitative study. Can J Surg 50:445-449, 2007

21. Hollenbeck BK, Dunn RL, Suskind AM, Zhang Y, Hollingsworth JM, Birkmeyer JD: Ambulatory surgery centers and outpatient procedure use among Medicare beneficiaries. Med Care 52:926-931, 2014

22. Hudak EM, Perry MW: Outpatient minimally invasive spine surgery using endoscopy for the treatment of lumbar spinal stenosis among obese patients. J Orthop 12:156-159, 2015

23. Idowu OA, Boyajian HH, Ramos E, Shi LL, Lee MJ: Trend of spine surgeries in the outpatient hospital setting versus ambulatory surgical center. Spine (Phila Pa 1976) 42:E1429-E1436, 2017

24. Khanna R, Kim RB, Lam SK, Cybulski GR, Smith ZA, Dahdaleh NS: comparing short-term complications of inpatient versus outpatient single-level anterior cervical discectomy and fusion: an analysis of 6940 patients using the ACS-NSQIP database. Clin Spine Surg 31:43-47, 2018

25. Lang SS, Chen HI, Koch MJ, Kurash L, McGill-Armento KR, Palella JM, et al: Development of an outpatient protocol for lumbar discectomy: our institutional experience. World Neurosurg 82:897-901, 2014

26. Lied B, Rønning PA, Halvorsen CM, Ekseth K, Helseth E: Outpatient anterior cervical discectomy and fusion for cervical disk disease: a prospective consecutive series of 96 patients. Acta Neurol Scand 127:31-37, 2013

27. Lied B, Sundseth J, Helseth E: Immediate (0-6 h), early (6-72 h) and late $(>72 \mathrm{~h})$ complications after anterior cervical discectomy with fusion for cervical disc degeneration; discharge six hours after operation is feasible. Acta Neurochir (Wien) 150:111-118, 2008

28. Martin BI, Deyo RA, Mirza SK, Turner JA, Comstock BA, Hollingworth W, et al: Expenditures and health status among adults with back and neck problems. JAMA 299:656-664, 2008 (Erratum in JAMA 299:2630, 2008)

29. McGirt MJ, Godil SS, Asher AL, Parker SL, Devin CJ: Quality analysis of anterior cervical discectomy and fusion in the outpatient versus inpatient setting: analysis of 7288 patients from the NSQIP database. Neurosurg Focus 39(6):E9, 2015

30. Missios S, Bekelis K: Hospitalization cost after spine surgery in the United States of America. J Clin Neurosci 22:16321637,2015

31. Mohandas A, Summa C, Worthington WB, Lerner J, Foley KT, Bohinski RJ, et al: Best practices for outpatient anterior cervical surgery: results from a Delphi panel. Spine (Phila Pa 1976) 42:E648-E659, 2017

32. Moses H III, Matheson DHM, Dorsey ER, George BP, Sadoff D, Yoshimura S: The anatomy of health care in the United States. JAMA 310:1947-1963, 2013

33. Nataraj A: Admission and acute complication rate for outpatient lumbar microdiscectomy. Can J Neurol Sci 37:1, 2010

34. Pugely AJ, Martin CT, Gao Y, Mendoza-Lattes SA: Outpatient surgery reduces short-term complications in lumbar discectomy: an analysis of 4310 patients from the ACS-NSQIP database. Spine (Phila Pa 1976) 38:264-271, 2013

35. Purger DA, Pendharkar AV, Ho AL, Sussman ES, Yang L, Desai M, et al: Outpatient vs inpatient anterior cervical discectomy and fusion: a population-level analysis of outcomes and cost. Neurosurgery [epub ahead of print], 2017 
36. Resnick DK, Tosteson ANA, Groman RF, Ghogawala Z: Setting the equation: establishing value in spine care. Spine (Phila Pa 1976) 39 (22 Suppl 1):S43-S50, 2014

37. Sheperd CS, Young WF: Instrumented outpatient anterior cervical discectomy and fusion: is it safe? Int Surg 97:8689,2012

38. Silvers HR, Lewis PJ, Suddaby LS, Asch HL, Clabeaux DE, Blumenson LE: Day surgery for cervical microdiscectomy: is it safe and effective? J Spinal Disord 9:287-293, 1996

39. Smith WD, Wohns RN, Christian G, Rodgers EJ, Rodgers WB: Outpatient minimally invasive lumbar interbody: fusion predictive factors and clinical results. Spine (Phila Pa 1976) 41 (Suppl 8):S106-S122, 2016

40. Stieber JR, Brown K, Donald GD, Cohen JD: Anterior cervical decompression and fusion with plate fixation as an outpatient procedure. Spine J 5:503-507, 2005

41. Techy F, Benzel EC: Implementing an outpatient ambulatory discectomy protocol at a large academic center: a change for the better. World Neurosurg 83:341-342, 2015

42. Trahan J, Abramova MV, Richter EO, Steck JC: Feasibility of anterior cervical discectomy and fusion as an outpatient procedure. World Neurosurg 75:145-148, 43-44, 2011

43. Villavicencio AT, Pushchak E, Burneikiene S, Thramann JJ: The safety of instrumented outpatient anterior cervical discectomy and fusion. Spine J 7:148-153, 2007

44. Walid MS, Robinson JS III, Robinson ERM, Brannick BB, Ajjan M, Robinson JS Jr: Comparison of outpatient and inpatient spine surgery patients with regards to obesity, comorbidities and readmission for infection. J Clin Neurosci 17:1497-1498, 2010
45. Wang MY: Outpatient anterior cervical discectomy and fusion. World Neurosurg 75:44, 2011

46. Wohns R: Safety and cost-effectiveness of outpatient cervical disc arthroplasty. Surg Neurol Int 1:77-74, 2010

47. Yen D, Albargi A: Results and limitations of outpatient and overnight stay laminectomies for lumbar spinal stenosis. Can J Surg 60:329-334, 2017

\section{Disclosures}

The authors report no conflicts of interest concerning the materials or methods used in this study or the findings specified in this paper.

\section{Author Contributions}

Conception and design: Pendharkar, Ho, Sussman, Purger, Veeravagu, Ratliff, Desai. Acquisition of data: Pendharkar, Shahin, Ho, Sussman, Purger, Veeravagu, Desai. Analysis and interpretation of data: all authors. Drafting the article: Pendharkar, Shahin, Ho, Sussman, Purger, Veeravagu, Desai. Critically revising the article: all authors. Reviewed submitted version of manuscript: all authors. Approved the final version of the manuscript on behalf of all authors: Pendharkar. Administrative/technical/material support: Pendharkar, Shahin, Ho.

\section{Correspondence}

Arjun Vivek Pendharkar: Stanford University School of Medicine, Stanford, CA. apendhar@stanford.edu. 